\title{
The influence of musical rhythm on a person's psychophysical state
}

\begin{abstract}
Background: In the modern world music is considered to be one of the strongest instruments in the sphere of medicine, psychology, pedagogy etc. The research of the music perception from the perspective of the biorhythms of a human body aligned to the corresponding acoustic rhythms would allow in the field of physical education to determine the level of influence of music on the quality of the realized educational, pedagogical, training, recovery and rehabilitation lessons. The aim of the research is to establish the influence of music on the psychophysical state of a person.
\end{abstract}

Methods: The following theoretical research methods were used: abstract an axiomatic method, analysis and synthesis, induction and deduction, idealization, comparison and generalization of materials on anatomy, physiology, psychophysiology, bio rhythmology, physics, biomechanics, music.

Results: In the given article are presented the rhythmic processes of perception of musical compositions that are different by structure and character in conformity with the human biorhythm. When they coincide a person at listening music receives positive emotions that arise the desire to live, create and develop. A negative influence is noticed at the perception of non-rhythmic sounds, which provokes in listeners either aggression of depression.

Conclusion: The physiological and psychological particularities of music must be taken into consideration both at the organization of the educational and pedagogical process of physical education, sport training, fitness and in the system of physical education lessons of recovery and rehabilitation.

Keywords: music, sound, rhythm, biorhythms, psychophysiology, education
Volume I Issue 3 - 2017

\author{
Aftimichuk Olga \\ State University of Physical Education and Sports, Moldova
}

Correspondence: Aftimichuk Olga, State University of Physical Education and Sports, Moldova, Email aftolig@mail.ru

Received: May 23, 2017 | Published: September II, 2017

\section{Introduction}

From the earliest times music was considered to be an important and irreplaceable means for the formation of personal qualities and inner world of a person. Even in Ancient Greece it was supported the idea that of music has an influence on the emotions of a person, it was proved that some melodies strengthen courage and endurance, others, on the contrary, make gentle. And in the modern world music is considered to be one of the strongest instruments in the sphere of medicine, psychology, pedagogy etc.

In opinion of such physiologists as NA Aladzhalova, ${ }^{1,2}$ NP Bekhtereva, ${ }^{3-5}$ VA Ilyukhina ${ }^{6,7}$ and others the human body is controlled by a super-slow rhythmic system. The basis of physiological perception of music is the deck seconds component of super-slow rhythmic processes, as the ones that control the processes of the human brain and entire body.

In our opinion, the research of the music perception from the perspective of the biorhythms of a human body aligned to the corresponding acoustic rhythms would allow in the field of physical education to determine the level of influence of music on the quality of the realized educational, pedagogical, training, recovery and rehabilitation lessons.

\section{Materials and methods of research}

The aim of the research is to establish the influence of music on the psychophysical state of a person. In accordance with this the tasks of the research are: study the phenomenon of music from the perspective of physics, physiology and psychology; determine the qualitative aspects of sound as an acoustic system; identify the correspondence between the biological and musical rhythmic characteristics. In the present work are analyzed the literature references on anatomy, ${ }^{8}$ physiology, ${ }^{9,10}$ psychophysiology, ${ }^{2,4,7}$ biorhythmology ${ }^{6,11}$ physics, ${ }^{12}$ mechanics, ${ }^{13}$ music. ${ }^{14-16}$ The realization of the set tasks presupposed the use of the following methods of theoretical research: abstract and axiomatic method, analysis and synthesis, induction and deduction, idealization, comparison and generalization.

\section{Results and discussion}

The basis of music is sound. As a physical phenomenon it is an oscillatory movement of a body, creating sound waves. The action of sound waves on the hearing organ through the acoustic nerve in the brain creates the perception of sound. Sounds are divided into musical and noise sounds. Unlike noise sounds, the musical sounds are selected and organized into a certain system, elaborated in the process of a century's long development of musical culture. To the group of musical sounds belong the sounds produced by different musical instruments, voices of animals and birds and also the human speech and singing. Sounds possess characteristics and qualities. Sound characteristics are its particularities: oscillation frequency, duration, amplitude and content (combination of simple oscillations in a more complicated unit). Sound quality, the reflection of above mentioned physical sound characteristics in our perception. To this group belong: height, duration, force (volume) and timbre (coloration). ${ }^{17}$ 
Height of sound depends on the frequency of sound oscillations. The more frequent is the oscillation - the higher is the sound, the less frequent is the oscillations - the lower is the sound. Pitch can be expressed with different levels of clarity. The sounds with a clear pitch are musical sounds, the ones without a clear pitch - noise sounds. The human hearing can perceive form 16 to 20000 oscillations per second. In music are basically used the sounds with a clear pitch within the range from 16 to 4000 oscillations per second. This is related to the practice of speech and singing. The speech itself uses sounds within even a closer limit. Sound volume depends on force of the oscillatory movement, expressed in the oscillation amplitude. The change of the sound volume during the execution of a musical composition is called dynamic inflection.

Timbre or color of the sound depends on the sound content. Each sound is not just a simple, but a combination of more tones which appear because the source of the sound oscillates not only as a whole, but at the same time some parts of it (half, third, fourth, fifth part etc.) which oscillates in its own way. Due to differences in timbre we distinguish one voice from another, the sound of one instrument from another, etc. Sound duration depends on the duration of the oscillatory movement. The organization of a sequence of similar or different sounds is called rhythm.

The influence of all musical and noise sounds is realized not only on the acoustic analyzer, but on the entire human body. This is related to the change of the dynamic characteristics of standing acoustic/ elastic waves in conformity with different ingredients of resonating characteristics of the human body and it manifests itself on the surface of the human body (boundary of body), by way of changing of slow potentials. It is worth mentioning, that unlike acoustic traveling waves, $99 \%$ of whose acoustic energy is absorbed on the skin surface, the acoustic perception of volume standing waves is perceived like something familiar, because, according to FK Agashin, ${ }^{12} 96 \mathrm{~ms}$ before this acoustic perception the acoustic wave through the water body structure already reaches the auditory cortex. The movement of the electrical impulse through neurons from the drum membranes to the auditory cortex is much slower (several tens of meters per second) than the hydraulic acoustic wave in the human body ( 1.5 kilometer per second). Thus, directly the body and namely its surface, perceives the sound much quicker than its hearing organ.

In her monograph "Psycho physiological aspects of super slow rhythmic activity of the human brain" NA Aladzhalova ${ }^{2}$ analyses the characteristics of "super slow rhythms" of the human brain and summaries them according to the following characteristics:

a. Spontaneity of the wide line rhythmic

b. Infra-low-frequency periodicity - appears as a new quality of the group union, as a collective form of participation at general regulations tasks

c. Cerebral, integrant, system process

d. Super-slow periodicity - acts as a factor of functional dynamics, on the basis of which lies the ability for flexible coordination, development of new forms of cooperation, which reflects the mechanisms that are at the basis of transformation of functional states, switch to new activity levels

e. stability, hierarchy principles of switching to new activity levels

f. Nonlinearity, auto regulation, self development.
However, for every type of rhythmic activity of brain there is a strictly determined corresponding psycho physiological state. NA Aladzhalova ${ }^{11}$ considers that the levels of consciousness are controlled by the slow rhythmic, the so-called "deck-seconds rhythm", which determines the form of change, structure of behavior of the fast speed rhythm system. Thus, the period of restless, emotional tension is 2-4 seconds, calm wakeful state of a person 5-10 seconds, the rhythm of falling into dormant state 360-40 seconds, the rhythm of passing form a conscious process to an unconscious one - 14 seconds. ${ }^{11}$

In this aspect the musical rhythm can be regarded both as means and condition of the assimilation of the given rhythm related to one or another physiological or psychological process. As it was presented above, music - is the organization in time mode of sounds, which embody artistic images. The expressive means of music are mode, rhythm, meter, dynamics (sound volume), register (pitch of sound), timbre, melody, harmony, polyphony etc. ${ }^{17}$ There is also the notion of musical syntax, which allows decomposing a piece of music into motive, phrase, sentence, period in conformity with the musical meter. Any type of music consists of singular, finished melodic phrases that constitute a musical period, which most fully reflects the natural biological super-slow rhythmic, both of the human brain and the entire body, which fact determines the psycho physiological norm of the body. ${ }^{10}$

The German chronobiologist $\mathrm{H}$ Pfalz ${ }^{15}$ made a correlation between the musical rhythm and memory for a musical composition on one hand and the biological rhythm of the human body on the other. For which reason he came to the conclusion that a person memorizes and likes exactly the musical compositions the rhythm of which mostly corresponds to his biological rhythm. Thus, the biological rhythms are, in a way, inner tuning forks for the perceived music and in the case when they coincide, then the person listens to music with pleasure. This music is better perceived and remembered. The negative influence on the body is identified at the perception of nonrhythmic, unstably repeating sounds, which are characteristic for the modern rock music. So, among the fans of "metallic rock" is attested a higher number of suicide attempts than among persons that prefer classic or religious music. ${ }^{16}$

The negative reaction of the body (initiation of a pathological state) is worsened by the deviation from the canonic melodic time and tone structures, "artificial" timbral content of electro musical instruments and especially musical computers. ${ }^{18}$ By this, at rock concerts there are cases of contusions caused by sound, acoustic combustions, hearing and memory loss; a distant effect can be the sleep disorder, because the sound intensity reaches 110-130 decibels (dB). According to normative acts, ${ }^{13}$ the levels of non-continuous noise for hospital and spa resort wards must be $35-45 \mathrm{~dB}$, living rooms $30-40 \mathrm{~dB}$, on the territory of residential constructions $45-55 \mathrm{~dB}$, railway stations and airports $60 \mathrm{~dB}$. The impact of industry related noise within $60-80 \mathrm{~dB}$ causes vegetative dysfunction in men, $90-110 \mathrm{~dB}$ - loss of hearing, 120 $\mathrm{dB}$ and more - deterioration of the auditory analyzer. ${ }^{19}$ The noise pain limit is on the level of $140 \mathrm{~dB}$, which can lead to lethal outcomes.

According to the data of hearing physiology, ${ }^{8}$ an important role in the increase of our ability to perceive sounds is played by the skin cover and bones of the skull. Reaching the external auditory canal sounds cause fluctuations of the drum membranes, which through the set of auditory ossicles are sent to the receiving structures of the internal ear. Besides this, the transmission of acoustic vibrations can be made not only by air. Thus, during singing with the mouth shut the sound is transmitted through skull bones directly to the internal ear. ${ }^{20}$ 
The sound is transmitted through soft tissues of the human body by means of the hydraulic wave (the body of an adult on the average consists of $70-75 \%$ water) or electromagnetic waves (industry related activity area of modern man). The impact on the entire body, but not only on the acoustic analyzer is possible due to the existence of an additional mechanism, which is not taken into consideration in neural models. ${ }^{18}$ The basic drawbacks of the neural model of information transmission from external stimuli is caused by the traditional presentation of the electrical conduction of nervous tissue, the interpretation of which is limited by the duration of reaction to external stimulation in $120-150 \mathrm{~ms}^{21}$ The non- accountable mechanism is related to the propagation through tissues of elastic, or acoustic waves, which program the neural answer in advance of the electric signal, as it as mentioned before, with $96 \mathrm{~ms}^{12}$

This advance, as VA Sinkevich ${ }^{18}$ considers, is related to the change of the dynamic characteristics of standing acoustic waves in conformity with the different ingredients of the resonating characteristics of the human body and it manifests itself on the surface as changes of the super-slow potential of the brain. This statement is taken into consideration for the realization of the psycho physiological researches in the field of music therapy.

According to the obtained data, sound can increase or decrease pain perception. This way, when having a toothache the sound of a waterfall has a positive influence. ${ }^{14}$ Besides this, it was established, that music therapy can be effective in decreasing the level of anxiety, blood pressure, pulse normalization, post operation pain, depression, vegetative dysfunctions. ${ }^{22,23}$ The results of the trans-cranial Doppler method (ultrasound research of the brain blood supply, which allows for the appreciation of the blood circulation through the intracranial vessels) had shown that the music relaxation therapy improves blood circulation. ${ }^{24}$

PP Moskalionov 9 had studied the influence of special natural acoustic compositions (elaborated by bioacoustics) on the vegetative, vessel and sensorial adaptation of the man and also he took up the appreciation of acoustic characteristics of music compositions, elaboration of methodological peculiarities in the use of computer stabilography for the appreciation of functional state of a person in normal state, when stressed and in an aggressive acoustic environment, appreciation of the influence of computer program of binaural stimulation on the stabilographic results of a person. In his researches the scientist comes to the following conclusions: ${ }^{9}$

I. The musical compositions elaborated by bioacoustics which include nature sounds and fragments of classical music; contribute to fatigue release and normalization of functional state of sensorial and cardiovascular systems.

II. Listening to musical compositions normalizes blood pressure, reduces the frequency of heart rate and increases the critical speed indicators, which fact is indicative of optimization of metabolic processes in cardiovascular system and visual analyzer.

III. It is indicated the use of frequency analysis for the appreciation of acoustic characteristics of musical compositions in ecological and rehabilitative sounds cape.

As a result of his research, PP Moskalionov ${ }^{9}$ had established that the binaural compositions, based on the physiological principles of acoustic analyzer functioning are an important impact factor for the somatic and psychological body state. In these circumstances the model binaural compositions can be used for stress release, performance and social communicability increase. For the purpose of general invigoration VA Sinkevich ${ }^{10}$ proposed the method of acoustic correction of functional state of the body, realized through the exposure to three dimensional acoustic fields within the limits of human hearing. The proposed acoustic stimulation includes a fragment of specially organized "white noise", which is reproduced simultaneously with the records of a human voice, some samples of classic music, ring of bells. Thus this music records are reproduced only with the help of a special acoustic system that creates running and standing acoustic waves which have an impact not only on the acoustic analyzer, but on the entire human body as well.

The research of VA Sinkevich ${ }^{10}$ is used under the title Musical and acoustic method of the noise therapy (MAMNT) and it is one of the universal and economically efficient methods of individual and group rehabilitation. According to the data collected by the author of the method during a month 8-12 half an hour sessions of MAMNT can bring a long - term improvement of the psycho physiological state of a person on the following positions:

i. Optimize the brain functions: memory, attention concentration; enlarge the auditory range of hard of hearing people etc.

ii. Relieve the majority of the types of psycho emotional stress; facilitate neurosis treatment, including the chronic fatigue syndrome, aggression of unclear etiology etc.

iii. Increase the working capacity, improve mood

iv. Normalize the "sleep-walk" cycle.

More than that, the above mentioned method, according to VA Sinkevich ${ }^{10}$ facilitates the treatment of such somatic diseases as functional disturbance of the prostate; menstruation normalization; relieves tic; optimizes kidney and gall bladder function; reduces the term of hematoma resolution and bones healing. Besides this, it facilitates treatment of such pathologies as post stress disorders; limbs traumas; cerebral commotions; enuresis; cardiovascular dysfunctions, logo neurosis; mental and psychological development delay; encephalopathy; neuro-circulatory asthenia and also it shortens the post operative care.

\section{Conclusion}

Consequently, the above presented materials of the specialized literature (anatomy, physiology, psychophysiology, biorhythmology, physics, biomechanics, music) and also the data of the realized researches in the field of physiology of hearing,, ${ }^{9,13}$ musical physiotherapy $^{22-24}$ state that the sound waves are a specific factor which universally influences the psychosomatic condition of a person. More than that, this influence can be either optimizing or pathogenic.

The present conclusion is important for the education sphere. On one hand, music is one of the education means, it is used for general education development of a person, on the other hand - it is used as a methodological means in the system of training sessions for a series of subjects ("Rhythmics", "Choreography", "Musical and rhythmic education", "Gymnastics", "Aerobics", "Sport dance" etc.). The teachers of the abovementioned subjects must be informed not only about the theory and methodology of music, but also about its psycho physiological particularities, because only this tandem will allow them to realize to the fullest the education process at any level. 


\section{Perspectives of further research}

In our opinion, the elaboration of the problem of professional training of specialists in physical culture is possible and challenging from the aspect of further researches of the music psychophysiology in the system of physical culture education for both didactic personnel and teaching methodology experts for therapeutic physical training, kinetic-therapists and sportsmen. The researches in this field presupposes a more qualitative and responsible approach towards the educational, recreational and rehabilitation processes, which fact would allow for the improvement of both the system of training of physical education teachers, sport/fitness coaches, teaching methodology experts for therapeutic physical training and the system of training lessons for any type of sport.

\section{Acknowledgements}

None.

\section{Conflict of interest}

Author declares there is no conflict of interest in publishing the article

\section{References}

1. Aladzhalova NA. Slow electrical processes in the brain. Elsevier Science, Netherlands; 1962. p. 242.

2. Aladzhalova NA. Psychophysiological aspects of the ultra-slow rhythmic activity of the brain. Science, Russia; 1979.

3. Bekhtereva NP. The magic of the brain and the labyrinth of life. 2nd edition. Revised and enlarged. Moscow-St. Petersburg, Russia; 2007.

4. Bekhtereva NP. The Usefulness of Psychophysiology in Intellectual Life. Honorary Lecture in $14^{\text {th }}$ World Congress of Psychophysiology. Russia; 2008 .

5. Benabid AL, Wallace B, Mitrofanis J, et al. Therapeutic electrical stimulation of the central nervous system. CR Biol. 2005;328(2):177-186.

6. Ilyukhina VA. Ultra-slow bioelectric processes of the human brain in the mechanisms of memory modulation. Human Physiology. 1981;7(3):512.

7. Ilyukhina VA. Neurophysiology of human functional states. Science. Russia; 1986.
8. Neiman LV, Bogomilsky MR. Anatomy, physiology and pathology of the organs of hearing and speech: VLADOS, Russia; 2001. p. 224.

9. Moskalionov PP. Ecological and physiological assessment of the influence of acoustic signals on human adaptation. Thesis CBS, Russia; 2008.

10. Sinkevich VA. Fractality of the natural "softness" of sound, light, heat. Foundations of new technology in the history of culture. Russia; 2010.

11. Aladzhalova NA. Rhythmic organization of mental and brain functions. Brain and mental activity. Science. Russia; 1984. p. 103-106.

12. Agashin FK. Biomechanics of shock movements. Physical Culture and Sports, Russia; 1977.

13. Ilyichev VD. Natural and technogenic noise the possibility of ecological comparisons by level. Proceedings of the Russian Academy of Sciences, Ser Biol. 1995;N3:351-360.

14. Good M. Effects of relaxation and music on postoperative pain: a review. J Adv Nurs. 1996;24(5):905-914.

15. Mezentsev VA. Miracles. The popular encyclopedia Vol 2. Home Edition of the Kazakh Soviet Encyclopedia - Alma-Ata, Russia; 1990. p. 370.

16. Stack S. Heavy metal, religiosity and suicide acceptability. Suicide Life Threat Behav. 1998;28(4):388-394.

17. Sposobin IV. Elementary theory of music. Music. Russia; 1964.

18. Sinkevich VA. The impact of a three-dimensional acoustic field on a person is a psychological aspect (musical psychotherapy). Volga Institute of Applied Psychology. Russia; 1992.

19. Kotova M. The Healing Sound. Science and Life. 1990;6:156-157.

20. Vartanyan IA. Sound - Hearing - Brain. Science. Russia; 1981.

21. Ivanitsky AM, Strelets VV, Korsakov IA. Information processes of the brain and mental activity. Nauka. Russia; 1984.

22. Manning J. Music therapy. BrJ Theatre Nurs. 1997;7(3):33-34.

23. Watkins GR. Music Therapy: proposed physiological mechanisms and clinical implications. Clin Nurse Spec. 1997;11(2):43-50.

24. Tang X, Yary F, Zhuany H. Musical relaxation therapy on the cerebral arteriosclerosis. Hunan Yi Ke Da Xue Xue Bao. 1997;22(1):45-48,52. 